УДК 517.9, 519.6

MSC 76M50, 80M40

\title{
HOMOGENIZED MODELS FOR MULTIPHASE DIFFUSION IN POROUS MEDIA
}

\section{G. V. SANDRAKOV}

Faculty of Computer Science and Cybernetics, Taras Shevchenko Kiev National University, Kiev, Ukraine, E-mail: gsandrako@gmail.com

\section{ОСРЕДНЕННЫЕ МОДЕЛИ МНОГОФАЗНОЙ ДИФФУЗИИ В ПОРИСТЫХ СРЕДАХ}

\section{Г. В. САНДРАКОВ}

Факультет компьютерных наук и кибернетики, Киевский национальный университет имени Тараса Шевченко, Киев, Украина, E-mail: gsandrako@gmail.com

ABSTRACT. Non-stationary processes of mutual diffusion for multiphase flows of immiscible liquids in porous media with a periodic structure are considered. The mathematical model for such processes is initial-boundary diffusion problem for media formed by a large number of «blocks» having low permeability and separated by a connected system of «cracks» with high permeability. Taking into account such a structure of porous media during modeling leads to the dependence of the equations of the problem on two small parameters of the porous medium microscale and the block permeability. Homogenized initial-boundary value problems will be obtained. Solutions of the problems are approximated for the solutions of the initial-boundary value problem under consideration.

KEYWORDS: multiphase diffusion, filtration processes, porous media, modeling, initial-boundary value problems, homogenized models.

АннотАция. Рассматриваются нестационарные процессы взаимной диффузии многофазных потоков несмешивающихся жидкостей в пористых средах с периодической структурой. Математической моделью для таких процессов является начально-краевая задача диффузии для сред, образованных большим количеством «блоков», имеющих слабую проницаемость, и разделенных связной системой «разломов» с высокой проницаемостью. Учет такого строения пористых сред приводит к зависимости уравнений этой задачи от двух малых параметров микромасштабности пористой среды и проницаемости блоков. Будут получены осредненные начально-краевые задачи, решения которых приближают решения рассматриваемой начально-краевой задачи.

КЛЮчЕВЫЕ СЛОВА: многофазная диффузия, процессы фильтрации, пористые среды, моделирование, начально-краевые задачи, осредненные модели. 


\section{ВвеДЕНИЕ}

Проблема моделирования нестационарных процессов взаимной диффузии и фильтрации многофазных потоков несмешивающихся жидкостей в пористых средах возникает при разработке месторождений нефти и газа, поиска методов предотвращения техногенных загрязнений (например, нефтепродуктами) в подземных водах и нахождения способов очистки таких вод от загрязнений. Непосредственное исследование таких процессов на практике техническими методами наблюдений практически не возможно, поскольку необходимо устанавливать большое количество датчиков на больших территориях и различных глубинах для понимания динамики совместного перемещения различных жидкостей в пористой среде. Таким образом, математическое моделирование является единственной возможность прогнозирования и возможной оптимизации методов нефтедобычи, газодобычи, очистки и предотвращения загрязнений подземных вод.

Достаточно подробная библиография об исследовании различных аспектов и методов применения математического и технического моделирования таких проблем на практике приведена в работах [1-6]. Однако, в этих работах практически не учитывается, что многие реальные пористые среды образованы большим количеством «блоков», имеющих слабую проницаемость, и разделенных связной системой «разломов» с высокой проницаемостью. Учет такого строений пористых сред при моделировании приводит к зависимости рассматриваемых моделей от двух параметров микромасштабности пористой среды и проницаемости блоков. Такие модели для однофазных уравнений с несколькими малыми параметрами и периодическими коэффициентами исследовались в работах [7-11]. Дальнейшие обобщения методов исследования этих моделей для задач диффузии и движения жидкостей, имеющих несколько фаз, приведено в работах [12-20]. При исследовании задач многофазной диффузии и фильтрации в пористых средах здесь будут использованы такие обобщения и метод осреднения задач диффузии, впервые предложенный в работах $[21,22]$ для задач, зависящих только от параметра микромасштабности.

\section{1. МОДЕЛЬ МНОГОФАЗНОЙ ДИФФУЗИИ В ПОРИСТЫХ СРЕДАХ}

Будут рассматриваться нестационарные процессы взаимной диффузии и фильтрации многофазных потоков несжимаемых несмешивающихся жидкостей в пористых средах с периодической структурой. В качестве математической модели для описания таких процессов принимается динамическая модель Маскета-Леверета для сред, образованных большим количеством «блоков», имеющих слабую проницаемость, и разделенных связной системой «разломов» с высокой проницаемостью. Данная модель является «типичной» для моделирования процессов газодобычи, нефтедобычи и диффузии загрязнений (например, нефтепродуктов) в подземных водах.

Допускается, что коэффициенты системы уравнений, характеризующих принятую модель, зависят от двух малых положительных параметров $\varepsilon$ и $\sigma$. Параметр мелко-масштабности $\varepsilon$ определяет период коэффициентов 
рассматриваемой системы уравнений, что соответствует предположению о периодической структуре рассматриваемых пористых сред, образованных блоками и разделенных хорошо проницаемыми разломами. Величина обратная к параметру $\sigma$, характеризует разброс коэффициентов тензора проницаемости, входящего в систему уравнений Маскета-Леверета, что соответствует предположению о слабой и высокой проницаемости блоков и разломов, образующих рассматриваемые пористые среды.

Предполагается, что при $\varepsilon \rightarrow 0$ выполнено соотношение $\sigma \rightarrow 0$ и одно из следующих трех условий, связывающих величины параметров периодичности и проницаемости:

$$
\begin{aligned}
\frac{\sigma}{\varepsilon^{2}} & \rightarrow \infty, \\
\frac{\sigma}{\varepsilon^{2}} & \rightarrow \vartheta, \\
\frac{\sigma}{\varepsilon^{2}} & \rightarrow 0,
\end{aligned}
$$

где $\vartheta$ обозначает заданную положительную постоянную. Таким образом, можно считать, что положительная постоянная $\sigma$ зависит от $\varepsilon$ и является достаточно малой при малых $\varepsilon$, а взаимная малость параметров $\varepsilon$ и $\sigma$ контролируется одним из предположений (1)-(3), выполняемым при $\varepsilon \rightarrow 0$.

Методами асимптотических разложений будут получены осредненная система уравнений и приведены теоремы о сходимости решений начальнокраевой задачи для системы уравнений Маскета-Леверета к решениям полученной осредненной системы. При построении асимптотических разложений и формулировках теорем о сходимости решений предполагается невырожденность рассматриваемой системы уравнений, что соответствует отсутствию «застойных зон» в процессе взаимной диффузии и фильтрации многофазных потоков в рассматриваемых пористых средах.

\section{2. НАЧАЛЬНО-КРАЕВЫЕ ЗАДАЧИ МНОГОФАЗНОЙ ДИФФУЗИИ}

Для точной формулировки конкретной начально-краевой задачи многофазной взаимной диффузии и фильтрации в пористой среде фиксируем ограниченную область $\Omega \subset \mathbb{R}^{n}$ для $n=2$ или $n=3$, положительные числа $\rho_{o}, \rho_{w}$ и $T$, вектор-функцию $G \in L^{2}\left(0, T ; L^{2}(\Omega)^{n}\right)$ и функции $G_{o}, G_{w} \in L^{2}\left(0, T ; L^{2}(\Omega)\right), S_{o}, S_{w} \in L^{\infty}(\Omega), P_{o}^{0}, P_{w}^{0} \in L^{2}\left(0, T ; H^{1}(\Omega)\right)$. Здесь и далее используются пространства функций, определения которых приведены, например, в [23]. Индексы $o$ и $w$ в обозначениях этих фиксированных функций означают, что эти функции относятся к одной из фаз рассматриваемых жидкостей, например, нефтепродуктов и воды.

Зададим функции $S_{o}^{\varepsilon}, S_{w}^{\varepsilon}, P_{o}^{\varepsilon}, P_{w}^{\varepsilon} \in L^{2}\left(0, T ; H^{1}(\Omega)\right)$, отвечающие объемным частям фаз и парциальным давлениям в этих фазах, как решения следующей начально-краевой задачи

$$
\begin{array}{ccc}
m_{\varepsilon}\left(S_{o}^{\varepsilon}\right)^{\prime}-\operatorname{div}\left(K_{\varepsilon}^{\sigma} \Lambda_{\varepsilon}^{o}\left(S_{o}^{\varepsilon}\right)\left[\nabla P_{o}^{\varepsilon}-\rho_{o} G\right]\right)=r_{\varepsilon}^{o} G_{o} & \text { в } & \Omega \times(0, T), \\
m_{\varepsilon}\left(S_{w}^{\varepsilon}\right)^{\prime}-\operatorname{div}\left(K_{\varepsilon}^{\sigma} \Lambda_{\varepsilon}^{w}\left(S_{w}^{\varepsilon}\right)\left[\nabla P_{w}^{\varepsilon}-\rho_{w} G\right]\right)=r_{\varepsilon}^{w} G_{w} & \text { в } \quad \Omega \times(0, T), \\
P_{w}^{\varepsilon}-P_{o}^{\varepsilon}=P_{\varepsilon}^{c}\left(S_{w}^{\varepsilon}\right) \quad \text { в } \quad \Omega \times(0, T), &
\end{array}
$$




$$
\begin{gathered}
S_{w}^{\varepsilon}+S_{o}^{\varepsilon}=1 \quad \text { в } \quad \Omega \times(0, T), \\
P_{w}^{\varepsilon}=P_{w}^{0}, \quad P_{o}^{\varepsilon}=P_{o}^{0} \quad \text { на } \partial \Omega \times(0, T), \\
\left.P_{w}^{\varepsilon}\right|_{t=0}=\left.P_{w}^{0}\right|_{t=0},\left.\quad P_{o}^{\varepsilon}\right|_{t=0}=\left.P_{o}^{0}\right|_{t=0} \quad \text { в } \quad \Omega, \\
\left.S_{w}^{\varepsilon}\right|_{t=0}=S_{w}^{0},\left.\quad S_{o}^{\varepsilon}\right|_{t=0}=S_{o}^{0} \quad \text { в } \quad \Omega .
\end{gathered}
$$

Таким образом, заданы четыре нестационарные уравнения с начальными и граничными условиями относительно четырех функций $S_{o}^{\varepsilon}, S_{w}^{\varepsilon}, P_{o}^{\varepsilon}$, $P_{w}^{\varepsilon} \in L^{2}\left(0, T ; H^{1}(\Omega)\right)$, которые корректно определены для фиксированных $\varepsilon$ и $\sigma$, что следует из результатов работ $[1,2]$ при условии невырожденности коэффициентов задачи (4). Однако, прежде следует привести зависимость коэффициентов задачи (4) от параметров $\varepsilon$ и $\sigma$ и определить условие невырожденности для таких коэффициентов. Для этого зададим сначала множества $F_{b}^{\varepsilon}$ и $F_{f}^{\varepsilon}$, соответствующие системам блоков и разломов, расположенных периодически с периодом $\varepsilon$.

Пусть $F_{f}$ является открытым связным 1-периодическим (периодическим с периодом 1 по каждой из независимых переменных $\left.x_{1}, \ldots, x_{n}\right)$ подмножеством из $\mathbb{R}^{n}$ с локально липшицевой границей, $F_{b}=\mathbb{R}^{n} \backslash F_{f}$ является множеством с локально липшицевой границей и

$$
F_{f}^{\varepsilon}=\varepsilon F_{f}=\left\{\varepsilon x: x \in F_{f}\right\}, \quad F_{b}^{\varepsilon}=\varepsilon F_{b}=\left\{\varepsilon x: x \in F_{b}\right\} .
$$

Таким образом, множества $F_{f}$ и $F_{b}$ с границами $\partial F_{f}$ и $\partial F_{b}$ вполне определяются множествами $Y_{f}=F_{f} \cap Y$ и $Y_{b}=F_{b} \cap Y$ с границами $\Gamma_{f}=\partial F_{f} \cap Y$ и $\Gamma_{b}=\partial F_{b} \cap Y$, где $Y=(0,1)^{n}$ обозначает ячейку периодичности множеств $F_{f}$ и $F_{b}$. Предполагается, что множества $Y_{f}$ и $Y_{b}$ имеют положительные меры Лебега в $\mathbb{R}^{n}$. Так определенные множества $Y_{f}$ и $Y_{b}$ разбивают ячейку периодичности $Y$ на два множества, соответствующих блокам и разломам, разделенными общими границами $\Gamma_{f}$ и $\Gamma_{b}$. Примеры, иллюстрирующие такие разбиения ячейки, приведены на рисунке 1.
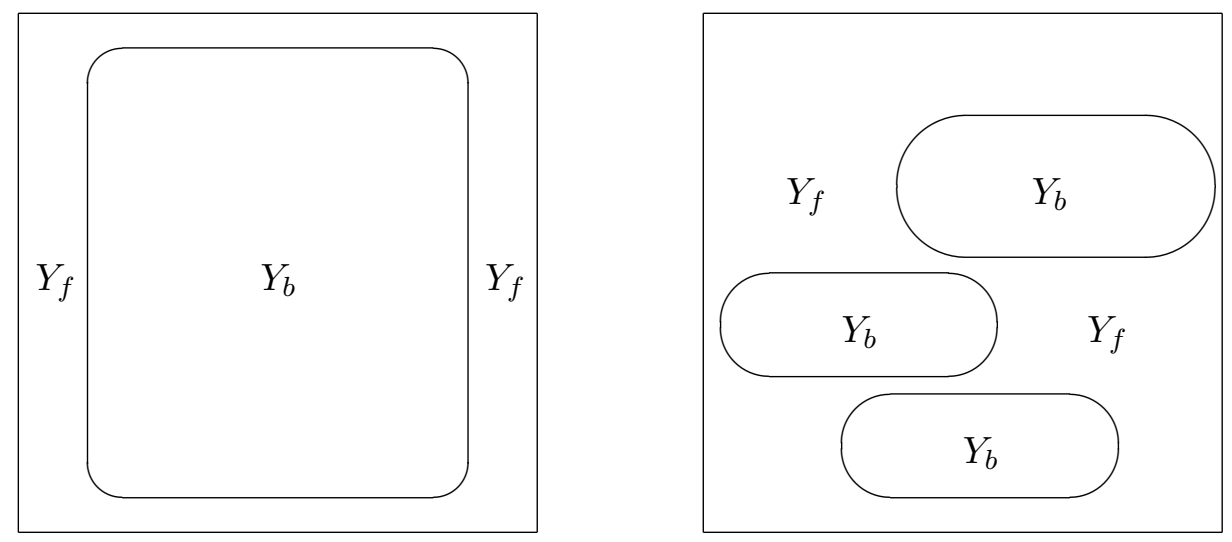

Рисунок 1. Модели разбиения ячейки на блоки и разломы 
Множества $F_{f}^{\varepsilon}$ и $F_{b}^{\varepsilon}$ для фиксированного $\varepsilon$ определяют естественным образом пористые среды с периодической структурой $\Omega_{b}^{\varepsilon}=F_{b}^{\varepsilon} \cap \Omega$ и $\Omega_{f}^{\varepsilon}=$ $F_{f}^{\varepsilon} \cap \Omega$, ограниченные границей $\partial \Omega$ множества $\Omega$, на котором и рассматривается задача (4). Примеры таких сред приведены на рисунке 2.
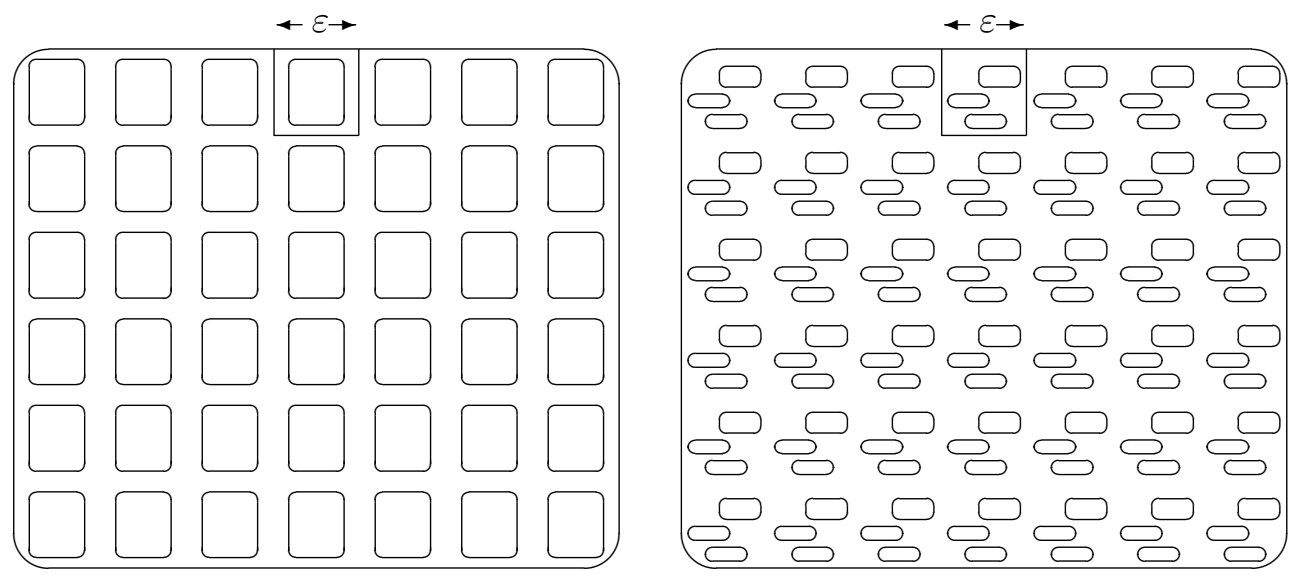

Рисунок 2. Модели пористых сред

Для так определенных моделей пористых сред $\Omega_{b}^{\varepsilon}=F_{b}^{\varepsilon} \cap \Omega$ и $\Omega_{f}^{\varepsilon}=F_{f}^{\varepsilon} \cap \Omega$, соответствующих блокам и разломам в области $\Omega$, зависимость коэффициентов задачи (4) от параметров $\varepsilon$ и $\sigma$ задается следующими равенствами

$$
\begin{gathered}
K_{\varepsilon}^{\sigma}=\sigma K_{b}(x / \varepsilon), m_{\varepsilon}=m_{b}(x / \varepsilon), \\
r_{\varepsilon}^{o}=r_{b}^{o}(x / \varepsilon), r_{\varepsilon}^{w}=r_{b}^{w}(x / \varepsilon) \text { в } \Omega_{b}^{\varepsilon}, \\
K_{\varepsilon}^{\sigma}=K_{f}(x / \varepsilon), m_{\varepsilon}=m_{f}(x / \varepsilon), \\
r_{\varepsilon}^{o}=r_{f}^{o}(x / \varepsilon), r_{\varepsilon}^{w}=r_{f}^{w}(x / \varepsilon) \text { в } \Omega_{f}^{\varepsilon} .
\end{gathered}
$$

Предполагается, что функции $m_{b}(y), r_{b}^{o}(y), r_{b}^{w}(y), m_{f}(y), r_{f}^{o}(y), r_{f}^{w}(y)$, и компоненты матричнозначных функций $K_{b}(y)$ и $K_{f}(y)$ являются ограниченными почти всюду функциями на множествах $F_{b}$ и $F_{f}$ соответственно. Кроме того, предполагается, что матричнозначные функции $K_{b}(y)$ и $K_{f}(y)$ являются симметричными и равномерно эллиптическими, а $m_{b}(y)$ и $m_{f}(y)$ отделены от нуля, что выражается следующими неравенствами

$$
\begin{gathered}
\alpha|\xi|^{2} \leq\left(K_{b}\left(y_{b}\right) \xi, \xi\right) \leq \beta|\xi|^{2}, \quad \alpha \leq m_{b}\left(y_{b}\right) \leq \beta, \\
\alpha|\xi|^{2} \leq\left(K_{f}\left(y_{f}\right) \xi, \xi\right) \leq \beta|\xi|^{2}, \quad \alpha \leq m_{f}\left(y_{f}\right) \leq \beta, \\
\text { для } y_{b} \in Y_{b}, \quad y_{f} \in Y_{f} \quad \text { и } \quad \xi \in \mathbb{R}^{n},
\end{gathered}
$$

где $\alpha$ и $\beta$ обозначают фиксированные положительные постоянные и $(\xi, \zeta)$ является скалярным произведением векторов $\xi, \zeta \in \mathbb{R}^{n}$.

Функции нелинейностей $\Lambda_{\varepsilon}^{o}, \Lambda_{\varepsilon}^{w}, P_{\varepsilon}^{c}$ в (4) зависят от $\varepsilon$ следующим образом

$$
\begin{aligned}
& \Lambda_{\varepsilon}^{o}=\Lambda_{b}^{o}(S), \quad \Lambda_{\varepsilon}^{w}=\Lambda_{b}^{w}(S), \quad P_{\varepsilon}^{c}=P_{b}^{c}(S) \quad \text { в } \quad \Omega_{b}^{\varepsilon}, \\
& \Lambda_{\varepsilon}^{o}=\Lambda_{f}^{o}(S), \quad \Lambda_{\varepsilon}^{w}=\Lambda_{f}^{w}(S), \quad P_{\varepsilon}^{c}=P_{f}^{c}(S) \quad \text { в } \quad \Omega_{f}^{\varepsilon}
\end{aligned}
$$


и заданы для $S \in[0,1]$ как функции нелинейностей для блоков и разломов, соответственно. Предполагается, что эти функции непрерывны и удовлетворяют следующим неравенствам невырожденности

$$
\begin{gathered}
\alpha \leq \Lambda_{b}^{o}(S)+\Lambda_{b}^{w}(S), \quad \alpha \leq \Lambda_{f}^{o}(S)+\Lambda_{f}^{w}(S), \\
\nu \leq \Lambda_{b}^{o}(S) \leq \beta, \quad \nu \leq \Lambda_{b}^{w}(S) \leq \beta \\
\nu \leq \Lambda_{f}^{o}(S) \leq \beta, \quad \nu \leq \Lambda_{f}^{w}(S) \leq \beta \quad \text { для } \quad S \in[0,1],
\end{gathered}
$$

где $\alpha, \beta$ и $\nu$ также обозначают фиксированные положительные постоянные.

Функции $P_{b}^{c}(S)$ и $P_{f}^{c}(S)$ из $(7)$ определяются как функции капиллярных давлений для соответствующих блоков и разломов и (по предположению) имеют некоторые неотрицательные первообразные

$$
\widetilde{P}_{b}^{c}(S)=\int^{S} P_{b}^{c}(\tau) d \tau \quad \text { и } \quad \widetilde{P}_{f}^{c}(S)=\int^{S} P_{f}^{c}(\tau) d \tau .
$$

Кроме того, предполагается, что функции $P_{b}^{-1}(S)$ и $P_{f}^{-1}(S)$, обратные к функциям $P_{b}^{c}(S)$ и $P_{f}^{c}(S)$ из $(7)$ и продолженные подходящим образом на всю прямую, являются липшицевыми и неубывающими на $\mathbb{R}$, имеющими производную всюду за исключением, возможно, конечного числа точек, и удовлетворяют следующим соотношениям

$$
\begin{gathered}
0 \leq P_{b}^{-1}(R) \leq 1, \quad 0 \leq P_{f}^{-1}(R) \leq 1 \quad \text { для } R \in \mathbb{R} \\
P_{b}^{-1}(0)=0, \quad P_{f}^{-1}(0)=0 .
\end{gathered}
$$

Относительно начальных и граничных данных для начально-краевой задачи (4) предполагается выполнение следующих условий

$$
\begin{gathered}
0 \leq S_{o}^{0} \leq 1, \quad 0 \leq S_{w}^{0} \leq 1, \quad S_{o}^{0}+S_{w}^{0}=1 \quad \text { в } \quad \Omega, \\
\left(P_{o}^{0}\right)_{t}^{\prime} \in L^{1}\left(0, T ; L^{2}(\Omega)\right), \quad\left(P_{w}^{0}\right)_{t}^{\prime} \in L^{1}\left(0, T ; L^{2}(\Omega)\right), \\
\int_{\Omega_{b}^{\varepsilon}} \widetilde{P}_{b}^{c}\left(S_{o}^{0}(x)\right) d \tau+\int_{\Omega_{f}^{\varepsilon}} \widetilde{P}_{f}^{c}\left(S_{o}^{0}(x)\right) d \tau \leq C,
\end{gathered}
$$

где постоянная $C$ не зависит от малого положительного параметра $\varepsilon$.

При сделанных предположениях начально-краевая задача (4) формулируется в вариационной форме или как задача сопряжения $[5,6]$, поскольку коэффициенты этой задачи являются разрывными в общем случае. Известно $[1,2]$, что для фиксированных $\sigma$ и $\varepsilon$ решение $S_{o}^{\varepsilon}, S_{w}^{\varepsilon}, P_{o}^{\varepsilon}$, $P_{w}^{\varepsilon} \in L^{2}\left(0, T ; H^{1}(\Omega)\right)$ задачи (4) существует. Функции $S_{o}^{\varepsilon}, S_{w}^{\varepsilon}, P_{o}^{\varepsilon}, P_{w}^{\varepsilon}$, задающие решение этой задачи, моделируют полную картину многофазной диффузии и фильтрации в рассматриваемой пористой среде. Однако, непосредственное нахождение этих функций вычислительными методами компьютерной математики практически не возможно, поскольку для рассматриваемых пористых сред (например, представленных на рисунке 2) необходимо выбирать очень мелкую сетку, зависящую от $\varepsilon$, чтобы точно учесть геометрию и конфигурации блоков и разломов.

В такой ситуации для более простых задач в работах $[21,22]$ был предложен следующий подход. Вначале находится приближенное решение к 
решению исходной задачи для малых $\varepsilon$. Такое приближенное решение удовлетворяет, обычно, начально-краевой задаче с постоянными коэффициентами, не зависящими от $\varepsilon$, рассматриваемой в области, также не зависящей от $\varepsilon$. Такую начально-краевую задачу с постоянными коэффициентами принято называть осредненной или осредненным приближением к исходной начально-краевой задаче, зависящей от дополнительных параметров, например, малого параметра $\varepsilon$. Далее, осредненная задача решается, как правило, стандартными вычислительными методами.

\section{3. ОСРЕДНЕННЫЕ ЗАДАЧИ МНОГОФАЗНОЙ ДИФФУЗИИ И ФИЛЬТРАЦИИ}

Для точной формулировки осредненных начально-краевых задач, решения которых приближают решения рассматриваемой задачи (4), понадобятся дополнительные определения и обозначения. Оказывается, что большая часть уравнений и начально-граничных условий осредненных задач не зависит от выполнения условий (1)-(3). Однако, уравнение распределения объемных фаз в блоках существенно зависит от выполнения этих условий. Коэффициенты осредненной задачи определяются следующим образом.

Пусть вектор-функция $N_{f}(y)$ является 1-периодическим решением следующей задачи Неймана на ячейке периодичности

$$
\begin{aligned}
& -\operatorname{div}_{y}\left(K_{f}(y) \nabla_{y} N_{f}(y)\right)=\operatorname{div}_{y}\left(K_{f}(y)\right) \quad \text { в } \quad Y_{f}, \\
& -\left(K_{f}(y) \nabla_{y} N_{f}(y), \Upsilon\right)=\left(K_{f}(y), \Upsilon\right) \quad \text { на } \quad \partial Y_{f},
\end{aligned}
$$

где $\Upsilon$ обозначает внешнюю нормаль к границе $\Gamma_{f}=\partial Y_{f}$.

Далее, учитывая определения (5), введем следующие обозначения для постоянных и матрицы с постоянными компонентами

$$
\begin{gathered}
\vartheta_{b}=\int_{Y_{b}} 1 d y, \quad \vartheta_{f}=\int_{Y_{f}} 1 d y \\
M_{b}=\int_{Y_{b}} m_{b}(y) d y, \quad M_{f}=\int_{Y_{f}} m_{f}(y) d y, \\
R_{o}=R_{b}^{o}+R_{f}^{o} \equiv \int_{Y_{b}} r_{b}^{o}(y) d y+\int_{Y_{f}} r_{f}^{o}(y) d y, \\
R_{w}=R_{b}^{w}+R_{f}^{w} \equiv \int_{Y_{b}} r_{b}^{w}(y) d y+\int_{Y_{f}} r_{f}^{w}(y) d y, \\
K=\int_{Y_{f}}\left(K_{f}(y)+K_{f}(y) \nabla_{y} N_{f}(y)\right) d y .
\end{gathered}
$$

Известно $[21,22]$, что матрица с постоянными компонентами $K$ является симметричной и и эллиптической. Определим функции $S_{o}^{f}, S_{o}^{b}, S_{w}^{f}, S_{w}^{b}$, $P_{o}, P_{w}$, отвечающие объемным частям фаз для разломов и блоков и парциальным давлениям в этих фазах, как решения следующей осредненной начально-краевой задачи

$$
\begin{gathered}
M_{f}\left(S_{o}^{f}\right)_{t}^{\prime}+M_{b}\left(S_{o}^{b}\right)_{t}^{\prime}-\operatorname{div}\left(K \Lambda_{f}^{o}\left(S_{o}^{f}\right)\left[\nabla P_{o}-\rho_{o} G\right]\right)=R_{o} G_{o} \text { в } \Omega \times(0, T), \\
M_{f}\left(S_{w}^{f}\right)_{t}^{\prime}+M_{b}\left(S_{w}^{b}\right)_{t}^{\prime}-\operatorname{div}\left(K \Lambda_{f}^{w}\left(S_{w}^{f}\right)\left[\nabla P_{w}-\rho_{w} G\right]\right)=R_{w} G_{w} \quad \text { в } \Omega \times(0, T),
\end{gathered}
$$




$$
\begin{gathered}
P_{w}-P_{o}=P_{f}^{c}\left(S_{w}^{f}\right) \quad \text { в } \quad \Omega \times(0, T), \\
S_{w}^{f}+S_{o}^{f}=1, \quad S_{w}^{b}+S_{o}^{b}=1 \quad \text { в } \quad \Omega \times(0, T), \\
P_{w}=P_{w}^{0}, \quad P_{o}=P_{o}^{0} \quad \text { на } \quad \partial \Omega \times(0, T), \\
\left.P_{w}^{\varepsilon}\right|_{t=0}=\left.P_{w}^{0}\right|_{t=0},\left.\quad P_{o}^{\varepsilon}\right|_{t=0}=\left.P_{o}^{0}\right|_{t=0} \quad \text { в } \quad \Omega, \\
\left.S_{w}^{f}\right|_{t=0}=S_{w}^{0},\left.\quad S_{o}^{f}\right|_{t=0}=S_{o}^{0} \quad \text { в } \quad \Omega, \\
\left.S_{w}^{b}\right|_{t=0}=S_{w}^{0},\left.\quad S_{o}^{b}\right|_{t=0}=S_{o}^{0} \quad \text { в } \quad \Omega .
\end{gathered}
$$

Таким образом, заданы пять нестационарных уравнений с начальными и граничными условиями относительно шести функций $S_{o}^{f}, S_{o}^{b}, S_{w}^{f}, S_{w}^{b}, P_{o}$, $P_{w}$, которые корректно определены, как выяснится далее, что будет следовать из приведенных ниже утверждений о сходимости решений $S_{o}^{\varepsilon}, S_{w}^{\varepsilon}$, $P_{o}^{\varepsilon}, P_{w}^{\varepsilon}$ исходной задачи (4). Формулировки этих утверждений зависят от выполнения одного из условий (1)-(3). При выполнении одного из этих условий выполняется и дополнительное шестое уравнение, которое замыкает осредненную систему уравнений (12), и гарантирует корректность определения функций $S_{o}^{f}, S_{o}^{b}, S_{w}^{f}, S_{w}^{b}, P_{o}, P_{w}$ как решений осредненной задачи.

Обозначим через $\chi_{b}^{\varepsilon}$ и $\chi_{f}^{\varepsilon}$ характеристические функции множеств $\Omega_{b}^{\varepsilon}$ и $\Omega_{f}^{\varepsilon}$. Следовательно, например, функции $\chi_{b}^{\varepsilon} P_{o}^{\varepsilon}$ и $\chi_{f}^{\varepsilon} P_{o}^{\varepsilon}$ обозначают ограничения функции парциального давления $P_{o}^{\varepsilon}$ на множества $\Omega_{b}^{\varepsilon}$ и $\Omega_{f}^{\varepsilon}$, моделирующих блоки и разломы пористой среды.

Для так определенных функций выполнено следующее утверждение.

Теорема 1. Пусть выполнено условие (1). Тогда найдутся такие подпоследовательности $S_{o}^{\varepsilon}, S_{w}^{\varepsilon}, P_{o}^{\varepsilon}, P_{w}^{\varepsilon}$ решений задачи (4), что

$$
\begin{array}{r}
\chi_{b}^{\varepsilon} S_{o}^{\varepsilon} \rightarrow S_{o}^{b}, \quad \chi_{f}^{\varepsilon} S_{o}^{\varepsilon} \rightarrow S_{o}^{f}, \quad \chi_{b}^{\varepsilon} S_{w}^{\varepsilon} \rightarrow S_{w}^{b}, \quad \chi_{f}^{\varepsilon} S_{w}^{\varepsilon} \rightarrow S_{w}^{f}, \\
\chi_{f}^{\varepsilon} P_{o}^{\varepsilon} \rightarrow \vartheta_{f} P_{o}, \quad \chi_{f}^{\varepsilon} P_{w}^{\varepsilon} \rightarrow \vartheta_{f} P_{w}
\end{array}
$$

$u$

$$
\chi_{b}^{\varepsilon} P_{o}^{\varepsilon} \rightarrow \vartheta_{b} P_{o}, \quad \chi_{b}^{\varepsilon} P_{w}^{\varepsilon} \rightarrow \vartheta_{b} P_{w}
$$

при $\varepsilon$, стремящемся к нулю, где функиии $S_{o}^{\varepsilon}, S_{w}^{\varepsilon}, P_{o}^{\varepsilon}, P_{w}^{\varepsilon} \in L^{2}\left(0, T ; L^{2}(\Omega)\right)$ удовлетворяют уравнениям и начально-краевым условиям задачи (12) и

$$
P_{w}-P_{o}=P_{b}^{c}\left(S_{w}^{b}\right) \quad \text { в } \quad \Omega \times(0, T) .
$$

Здесь и в дальнейшем, обозначения $\rightarrow$ и $\rightarrow$ соответствуют сильной и слабой сходимости в функциональном пространстве $L^{2}\left(0, T ; L^{2}(\Omega)\right)$.

Пусть дополнительные функции $Q(y), Q_{o}(y), Q_{w}(y)$ являются 1-периодическими решениями следующих задач Дирихле

$$
\begin{array}{ccccccc}
-\operatorname{div}_{y}\left(K_{b}(y) \nabla_{y} Q(y)\right)=m_{b}(y) & \text { в } & Y_{b}, & Q=0 & \text { на } & Y_{f}, \\
-\operatorname{div}_{y}\left(K_{b}(y) \nabla_{y} Q_{o}(y)\right)=r_{b}^{o}(y) & \text { в } & Y_{b}, & Q_{o}=0 & \text { на } & Y_{f}, \\
-\operatorname{div}_{y}\left(K_{b}(y) \nabla_{y} Q_{w}(y)\right)=r_{w}^{o}(y) & \text { в } & Y_{b}, & Q_{w}=0 & \text { на } & Y_{f} .
\end{array}
$$

Используя решения этих задач, которые определены однозначно, введем постоянные

$$
\vartheta_{m}=\int_{Y_{b}} Q d x, \quad \vartheta_{o}^{r}=\int_{Y_{b}} Q_{o} d x, \quad \vartheta_{w}^{r}=\int_{Y_{b}} Q_{w} d x
$$


Из принципа максимума для задачи Дирихле и предположений (6) следует, что постоянная $\vartheta_{m}$ всегда положительна.

Кроме того, выполнены следующие утверждения.

Теорема 2. Пусть выполнено условие (2). Тогда найдутся такие подпоследовательности $S_{o}^{\varepsilon}, S_{w}^{\varepsilon}, P_{o}^{\varepsilon}, P_{w}^{\varepsilon}$ решений задачи (4), что выполнены соотношения (13) и

$$
\begin{gathered}
\chi_{b}^{\varepsilon} P_{o}^{\varepsilon} \rightarrow \vartheta_{b} P_{o}+\frac{\vartheta_{o}^{r} G_{o}}{\vartheta \Lambda_{b}^{o}\left(S_{o}^{b}\right)}-\frac{\vartheta_{m}\left(S_{o}^{b}\right)_{t}^{\prime}}{\vartheta \Lambda_{b}^{o}\left(S_{o}^{b}\right)}, \\
\chi_{b}^{\varepsilon} P_{w}^{\varepsilon} \rightarrow \vartheta_{b} P_{w}+\frac{\vartheta_{w}^{r} G_{w}}{\vartheta \Lambda_{b}^{w}\left(S_{w}^{b}\right)}-\frac{\vartheta_{m}\left(S_{w}^{b}\right)_{t}^{\prime}}{\vartheta \Lambda_{b}^{w}\left(S_{w}^{b}\right)}
\end{gathered}
$$

при є, стремящемся к нулю, где функиии $S_{o}^{\varepsilon}, S_{w}^{\varepsilon}, P_{o}^{\varepsilon}, P_{w}^{\varepsilon} \in L^{2}\left(0, T ; L^{2}(\Omega)\right)$ удовлетворяют уравнениям и начально-краевым условиям задачи (12) и в области $\Omega \times(0, T)$ уравнению

$$
\begin{gathered}
\vartheta_{m}\left(\Lambda_{b}^{w}\left(S_{w}^{b}\right)+\Lambda_{b}^{o}\left(S_{o}^{b}\right)\right)\left(S_{w}^{b}\right)_{t}^{\prime}= \\
=\vartheta_{b} \vartheta \Lambda_{b}^{w}\left(S_{w}^{b}\right) \Lambda_{b}^{o}\left(S_{w}^{b}\right)\left(P_{f}^{c}\left(S_{w}^{f}\right)-P_{b}^{c}\left(S_{w}^{b}\right)\right)+\vartheta_{w}^{r} G_{w} \Lambda_{b}^{o}\left(S_{o}^{b}\right)-\vartheta_{o}^{r} G_{o} \Lambda_{b}^{w}\left(S_{w}^{b}\right) .
\end{gathered}
$$

Теорема 3. Пусть выполнено условие (3). Тогда найдутся такие подпоследовательности $S_{o}^{\varepsilon}, S_{w}^{\varepsilon}, P_{o}^{\varepsilon}, P_{w}^{\varepsilon}$ решений задачи (4), что выполнены соотношения (13) и

$$
\begin{aligned}
& \left(\sigma / \varepsilon^{2}\right) \chi_{b}^{\varepsilon} P_{o}^{\varepsilon}-\frac{\vartheta_{o}^{r} G_{o}}{\Lambda_{b}^{o}\left(S_{o}^{b}\right)}-\frac{\vartheta_{m}\left(S_{o}^{b}\right)_{t}^{\prime}}{\Lambda_{b}^{o}\left(S_{o}^{b}\right)}, \\
& \left(\sigma / \varepsilon^{2}\right) \chi_{b}^{\varepsilon} P_{w}^{\varepsilon}-\frac{\vartheta_{w}^{r} G_{w}}{\Lambda_{b}^{w}\left(S_{w}^{b}\right)}-\frac{\vartheta_{m}\left(S_{w}^{b}\right)_{t}^{\prime}}{\Lambda_{b}^{w}\left(S_{w}^{b}\right)}
\end{aligned}
$$

при $\varepsilon$, стремящемся к нулю, где функи,ии $S_{o}^{\varepsilon}, S_{w}^{\varepsilon}, P_{o}^{\varepsilon}, P_{w}^{\varepsilon} \in L^{2}\left(0, T ; L^{2}(\Omega)\right)$ удовлетворяют уравнениям и начально-краевым условиям задачи (12) при $M_{b}=R_{b}^{o}=R_{b}^{w}=0$ и в области $\Omega \times(0, T)$ уравнению

$$
\vartheta_{m}\left(\Lambda_{b}^{w}\left(S_{w}^{b}\right)+\Lambda_{b}^{o}\left(S_{o}^{b}\right)\right)\left(S_{w}^{b}\right)_{t}^{\prime}=\vartheta_{w}^{r} G_{w} \Lambda_{b}^{o}\left(S_{o}^{b}\right)-\vartheta_{o}^{r} G_{o} \Lambda_{b}^{w}\left(S_{w}^{b}\right) .
$$

Последнее равенство при $\vartheta_{w}^{r}=0$ и $\vartheta_{o}^{r}=0$ вместе с равенством $S_{w}^{b}+S_{o}^{b}=1$ из $(12)$ означают, что функции $S_{w}^{b}$ и $S_{o}^{b}$, отвечающие ограничениям объемным частей фаз на систему блоков пористой среды, стационарны и не изменяются со временем при отсутствии внешних источников. Этот вывод является естественным, поскольку при выполнении условия (3) блоки являются очень слабо проницаемыми, например, можно выбрать $\sigma=\varepsilon^{3}$.

В случае выполнения условий (1) и (2) ситуация значительно сложнее, поскольку необходимо рассматривать ограничения объемных частей фаз и на систему блоков и на систему разломов. Осредненная система уравнений (12) моделирующая динамику этих ограничений является в некотором смысле «удвоением» исходной системы вместе с равенством (15), если выполнено условие (1). При выполнении условий (2) или (3) такое «удвоение» является достаточно сложным в силу уравнений (17) и (19). 
Важно подчеркнуть, что во всех случаях осредненные системы уравнений не зависят от малых параметров $\varepsilon$ и $\sigma$, характеризующих микромасштабность пористой среды и слабость проницаемости блоков.

Таким образом, приближенные численные решения этих осредненных систем уравнений могут быть найдены непосредственно с помощью стандартных вычислительных методов компьютерной математики в области $\Omega$, не зависящей от малых параметров.

Приближенные асимптотические разложения решений рассматриваемой задачи (4) находятся методами работ [7-11], где рассматривались начальнокраевые задачи для линейных уравнений, зависящие от трех малых параметров. После того как начальные слагаемые асимптотических разложений построены, доказательства теорем 1-3 проводиться стандартными энергетическими методами. В процессе такого доказательства устанавливается и существование (по крайней мере одного) решения соответствующей осредненной системы уравнений. Кроме того, при выполнении одного из условий теорем 1-3 подпоследовательности $\chi_{b}^{\varepsilon} S_{o}^{\varepsilon}, \chi_{f}^{\varepsilon} S_{o}^{\varepsilon}, \chi_{b}^{\varepsilon} S_{w}^{\varepsilon}$ и $\chi_{f}^{\varepsilon} S_{w}^{\varepsilon}$ сходятся и почти всюду в $\Omega \times(0, T)$. Поэтому для предельных функций $S_{o}^{b}, S_{o}^{f}, S_{w}^{b}$ и $S_{w}^{f}$ сохраняются неравенства неотрицательности и ограниченности

$$
0 \leq \chi_{b}^{\varepsilon} S_{o}^{\varepsilon} \leq 1, \quad 0 \leq \chi_{f}^{\varepsilon} S_{o}^{\varepsilon} \leq 1, \quad 0 \leq \chi_{b}^{\varepsilon} S_{w}^{\varepsilon} \leq 1, \quad 0 \leq \chi_{f}^{\varepsilon} S_{w}^{\varepsilon} \leq 1,
$$

выполненные почти всюду в $\Omega \times(0, T)$ для решений задачи (4).

Предположения (8) и (9) на функции нелинейностей в задаче (4) являются стандартными, за исключением условия положительности постоянной $\nu$ из (8). Физический интерес представляет и вырожденный случай, когда $\nu=0$. Обычно в этом случае задача (4) преобразуется к приведенной задаче (которая определена, например, в $[5,6]$ ). Однако такое преобразование существенно нелинейно и не всегда ясно, в каком смысле исходная и приведенная задачи эквивалентны.

Известно [1, 2], что и для вырожденной задачи (4) неравенства (8) выполняются, если начальные и граничные данные в этой задаче не генерируют вырождения. В случае, когда на некоторой части границы $\partial \Omega \times(0, T)$ заданы неоднородные условия Неймана и рассматривается вырожденная задача, для выполнения неравенств (8) необходимы дополнительные предположения на порядок функций нелинейностей [2]. В предположении выполнения неравенств (8) соответствующие осредненные системы уравнений остаются неизменными и для начально-краевой задачи Дирихле-Неймана.

\section{4. АСИМПТОТИЧЕСКИЕ РАЗЛОЖЕНИЯ РЕШЕНИЙ ЗАДАЧИ ДИФФУЗИИ}

Алгоритм построения начальных асимптотических разложений решений общей нестационарной системы уравнений упругости второго порядка с быстро осциллирующими контрастными коэффициентами подробно описан в работе [8]. В соответствии с этими построениями асимптотическое приближение к решению задачи (4) можно выбрать в виде

$$
\begin{aligned}
& S_{o}^{a}=S_{o}(t, x)+\varepsilon R_{o}(t, x, x / \varepsilon), \quad S_{w}^{a}=S_{w}(t, x)+\varepsilon R_{w}(t, x, x / \varepsilon), \\
& P_{o}^{a}=P_{o}(t, x)+V_{o}(t, x, x / \varepsilon)+\varepsilon U_{o}(t, x, x / \varepsilon)+\varepsilon^{2} W_{o}(t, x, x / \varepsilon),
\end{aligned}
$$




$$
P_{w}^{a}=P_{w}(t, x)+V_{w}(t, x, x / \varepsilon)+\varepsilon U_{w}(t, x, x / \varepsilon)+\varepsilon^{2} W_{w}(t, x, x / \varepsilon),
$$

где функции $R_{o}(t, x, y), R_{w}(t, x, y), \ldots, W_{o}(t, x, y), W_{w}(t, x, y)$ являются 1-периодическими по переменной $y \in \mathbb{R}^{n}$. Будем также обозначать через

$$
S_{o}^{b \varepsilon}=\chi_{b}^{\varepsilon} S_{o}^{\varepsilon}(x), \ldots, P_{w}^{f \varepsilon}=\chi_{f}^{\varepsilon} P_{w}^{\varepsilon}(x)
$$

ограничения функций $S_{o}^{\varepsilon}(x), \ldots, P_{w}^{\varepsilon}(x)$ на множества $\Omega_{b}^{\varepsilon}$ и $\Omega_{f}^{\varepsilon}$, соответственно. Кроме того, в равенствах (20) предполагается, что

$$
V_{o}(t, x, x / \varepsilon)=0, \quad V_{w}(t, x, x / \varepsilon)=0 \quad \text { для } \quad x \in \Omega_{f}^{\varepsilon} \times(0, T) .
$$

Следуя [22], задачу (4) можно записать в следующем эквивалентном виде

$$
\begin{aligned}
& m_{b}^{\varepsilon}\left(S_{o}^{b \varepsilon}\right)^{\prime}-\sigma \operatorname{div}\left(K_{b}^{\varepsilon} \Lambda_{b}^{o}\left(S_{o}^{b \varepsilon}\right)\left[\nabla P_{o}^{b \varepsilon}-\rho_{o} G\right]\right)=r_{b}^{o \varepsilon} G_{o} \quad \text { в } \quad \Omega_{b}^{\varepsilon} \times(0, T), \\
& m_{f}^{\varepsilon}\left(S_{o}^{f \varepsilon}\right)^{\prime}-\operatorname{div}\left(K_{f}^{\varepsilon} \Lambda_{f}^{o}\left(S_{o}^{f \varepsilon}\right)\left[\nabla P_{o}^{f \varepsilon}-\rho_{o} G\right]\right)=r_{f}^{o \varepsilon} G_{o} \quad \text { в } \Omega_{f}^{\varepsilon} \times(0, T), \\
& m_{b}^{\varepsilon}\left(S_{w}^{b \varepsilon}\right)^{\prime}-\sigma \operatorname{div}\left(K_{b}^{\varepsilon} \Lambda_{b}^{w}\left(S_{w}^{b \varepsilon}\right)\left[\nabla P_{w}^{b \varepsilon}-\rho_{w} G\right]\right)=r_{b}^{w \varepsilon} G_{o} \quad \text { в } \quad \Omega_{b}^{\varepsilon} \times(0, T), \\
& m_{f}^{\varepsilon}\left(S_{w}^{f \varepsilon}\right)^{\prime}-\operatorname{div}\left(K_{f}^{\varepsilon} \Lambda_{f}^{w}\left(S_{w}^{f \varepsilon}\right)\left[\nabla P_{w}^{f \varepsilon}-\rho_{w} G\right]\right)=r_{f}^{w \varepsilon} G_{o} \quad \text { в } \quad \Omega_{f}^{\varepsilon} \times(0, T)
\end{aligned}
$$

совместно со следующими граничными условиями сопряжения на общей границе областей $\Omega_{f}^{\varepsilon}$ и $\Omega_{b}^{\varepsilon}$

$$
\begin{gathered}
\left(K_{f}^{\varepsilon} \Lambda_{f}^{o}\left(S_{o}^{f \varepsilon}\right)\left[\nabla P_{o}^{f \varepsilon}-\rho_{o} G\right], \Upsilon_{\varepsilon}\right)= \\
=\sigma\left(K_{b}^{\varepsilon} \Lambda_{b}^{o}\left(S_{o}^{b \varepsilon}\right)\left[\nabla P_{o}^{b \varepsilon}-\rho_{o} G\right], \Upsilon_{\varepsilon}\right) \quad \text { на } \Theta_{\varepsilon} \times(0, T), \\
\left(K_{f}^{\varepsilon} \Lambda_{f}^{w}\left(S_{w}^{f \varepsilon}\right)\left[\nabla P_{w}^{f \varepsilon}-\rho_{w} G\right], \Upsilon_{\varepsilon}\right)= \\
=\sigma\left(K_{b}^{\varepsilon} \Lambda_{b}^{w}\left(S_{w}^{b \varepsilon}\right)\left[\nabla P_{w}^{b \varepsilon}-\rho_{w} G\right], \Upsilon_{\varepsilon}\right) \quad \text { на } \Theta_{\varepsilon} \times(0, T),
\end{gathered}
$$

где, например, $\Theta_{\varepsilon}=\partial \Omega_{f}^{\varepsilon} \backslash \partial \Omega$ обозначает общую границу областей $\Omega_{f}^{\varepsilon}$ и $\Omega_{b}^{\varepsilon}$ с внешней нормалью $\Upsilon_{\varepsilon}, m_{b}^{\varepsilon}=m_{b}(x / \varepsilon), \ldots, K_{f}^{\varepsilon}=K_{f}(x / \varepsilon)$ и остальные уравнения задачи (4) остаются неизменными.

Для построения асимптотических разложений (20) для решений задачи (4), следующие две формулы для функций вида $V(x, x / \varepsilon)$, зависящих от $\varepsilon$ специальным образом, будут использоваться в дальнейшем

$$
\begin{aligned}
\nabla(V(x, x / \varepsilon)) & =\left.\left(\varepsilon^{-1} \nabla_{y} V(x, y)+\nabla_{x} V(x, y)\right)\right|_{y=x / \varepsilon}, \\
\operatorname{div}(V(x, x / \varepsilon)) & =\left.\left(\varepsilon^{-1} \operatorname{div}_{y} V(x, y)+\operatorname{div}_{x} V(x, y)\right)\right|_{y=x / \varepsilon} .
\end{aligned}
$$

Подставим разложения (20) в уравнения из $(21)$ на множестве $\Omega_{f}^{\varepsilon} \times(0, T)$. Тогда, учитывая равенства (23) и сохраняя только существенные для дальнейшего слагаемые, получаем

$$
\begin{gathered}
m_{f}\left(S_{o}^{f}\right)^{\prime}-\varepsilon^{-1} \operatorname{div}_{y}\left(K_{f} \Lambda_{f}^{o}\left(S_{o}^{f}\right) \nabla_{y} U_{o}^{f}\right)- \\
-\varepsilon^{-1} \operatorname{div}_{y}\left(K_{f} \Lambda_{f}^{o}\left(S_{o}^{f}\right)\left[\nabla P_{o}^{f}-\rho_{o} G\right]\right)-\operatorname{div}_{y}\left(K_{f} \Lambda_{f}^{o}\left(S_{o}^{f}\right) \nabla_{y} W_{o}^{f}\right)- \\
-\operatorname{div}_{x}\left(K_{f} \Lambda_{f}^{o}\left(S_{o}^{f}\right) \nabla_{y} U_{o}^{f}\right)-\operatorname{div}_{x}\left(K_{f} \Lambda_{f}^{o}\left(S_{o}^{f}\right)\left[\nabla P_{o}^{f}-\rho_{o} G\right]\right)- \\
-\operatorname{div}_{y}\left(K_{f} \Lambda_{f}^{o}\left(S_{o}^{f}\right) \nabla_{x} U_{o}^{f}\right)=r_{f}^{o} G_{o} \quad \text { при } y=x / \varepsilon \text { и } x \in \Omega_{f}^{\varepsilon} \times(0, T) .
\end{gathered}
$$

Аналогичное соотношение для функций $S_{w}^{f}, U_{w}^{f}, W_{w}^{f}$ и $P_{w}^{f}$ из асимптотического разложения (20) имеет следующий вид

$$
m_{f}\left(S_{w}^{f}\right)^{\prime}-\varepsilon^{-1} \operatorname{div}_{y}\left(K_{f} \Lambda_{f}^{w}\left(S_{w}^{f}\right) \nabla_{y} U_{w}^{f}\right)-
$$




$$
\begin{gathered}
-\varepsilon^{-1} \operatorname{div}_{y}\left(K_{f} \Lambda_{f}^{w}\left(S_{w}^{f}\right)\left[\nabla P_{w}^{f}-\rho_{w} G\right]\right)-\operatorname{div}_{y}\left(K_{f} \Lambda_{f}^{w}\left(S_{w}^{f}\right) \nabla_{y} W_{w}^{f}\right)- \\
-\operatorname{div}_{x}\left(K_{f} \Lambda_{f}^{w}\left(S_{w}^{f}\right) \nabla_{y} U_{w}^{f}\right)-\operatorname{div}_{x}\left(K_{f} \Lambda_{f}^{w}\left(S_{w}^{f}\right)\left[\nabla P_{w}^{f}-\rho_{w} G\right]\right)- \\
-\operatorname{div}_{y}\left(K_{f} \Lambda_{f}^{w}\left(S_{w}^{f}\right) \nabla_{x} U_{w}^{f}\right)=r_{f}^{w} G_{w} \quad \text { при } y=x / \varepsilon \text { и } x \in \Omega_{f}^{\varepsilon} \times(0, T) .
\end{gathered}
$$

Из этого соотношения и соотношения (24) будет получена осредненная начально-краевая задача (12), точная форма которой зависит от выполнения одного из условий (1)-(3). Таким образом, следует рассматривать эти случаи отдельно для каждого из условий (1)-(3).

\section{5. ОБОСНОВАНИЕ АСИМПТОТИЧЕСКИХ РАЗЛОЖЕНИЙ РЕШЕНИЙ}

Пусть выполнено условие (2). Тогда разность $\delta=\vartheta-\sigma / \varepsilon^{2}$ можно считать малым параметром. Подставляя разложения (20) в условия сопряжения (22) и сохраняя только существенные слагаемые, получаем

$$
\begin{gathered}
\left(K_{f} \Lambda_{f}^{o}\left(S_{o}^{f}\right)\left[\nabla_{y} U_{o}^{f}+\nabla P_{o}^{f}-\rho_{o} G+\varepsilon \nabla_{y} W_{o}^{f}+\varepsilon \nabla_{x} U_{o}^{f}\right], \Upsilon_{\varepsilon}\right)= \\
=\varepsilon \vartheta\left(K_{b} \Lambda_{b}^{o}\left(S_{o}^{b}\right)\left[\nabla_{y} V_{o}^{b}+\varepsilon\left(\nabla P_{o}^{b}-\rho_{o} G\right)+\varepsilon \nabla_{y} U_{o}^{b}\right], \Upsilon_{\varepsilon}\right)- \\
-\varepsilon \delta\left(K_{b} \Lambda_{b}^{o}\left(S_{o}^{b}\right)\left[\nabla_{y} V_{o}^{b}+\ldots\right], \Upsilon_{\varepsilon}\right) \quad \text { при } y=x / \varepsilon \text { и } x \in \Theta_{\varepsilon} \times(0, T) .
\end{gathered}
$$

Приравнивая коэффициенты при $\varepsilon^{-1}$ в (24) и при $\varepsilon^{0}$ в $(25)$, имеем

$$
\begin{aligned}
& -\operatorname{div}_{y}\left(K_{f} \Lambda_{f}^{o}\left(S_{o}^{f}\right) \nabla_{y} U_{o}^{f}\right)=\operatorname{div}_{y}\left(K_{f} \Lambda_{f}^{o}\left(S_{o}^{f}\right)\left[\nabla P_{o}^{f}-\rho_{o} G\right]\right), \\
& -\left(K_{f} \Lambda_{f}^{o}\left(S_{o}^{f}\right) \nabla_{y} U_{o}^{f}, \Upsilon_{\varepsilon}\right)=\left(K_{f} \Lambda_{f}^{o}\left(S_{o}^{f}\right)\left[\nabla P_{o}^{f}-\rho_{o} G\right], \Upsilon_{\varepsilon}\right) .
\end{aligned}
$$

Эти равенства будут выполнены, если выбрать

$$
U_{o}^{f}=N_{f}(y)\left[\nabla P_{o}^{f}(t, x)-\rho_{o} G(t, x)\right],
$$

где $N_{f}(y)$ определяется однозначно (условием $\int_{Y_{f}} N_{f}(y) d y=0$ ) как решение 1-периодической задачи Неймана, приведенной перед соотношениями (11). Аналогично, получаем равенство

$$
U_{w}^{f}=N_{f}(y)\left[\nabla P_{w}^{f}(t, x)-\rho_{w} G(t, x)\right] .
$$

Продолжим функцию $N_{f}(y)$ на $Y_{b}$ как 1-периодическое решение задачи

$$
\operatorname{div}_{y}\left(K_{b} \nabla_{y} N_{f}\right)=0 \quad \text { в } \quad Y_{b}, \quad N_{f}=0 \quad \text { в } \quad \partial Y_{b}
$$

и обозначим полученную функцию, определенную однозначно на ячейке периодичности $Y$, через $N(y)$. Тогда можно выбрать

$$
\begin{aligned}
U_{o} & =N(y)\left[\nabla P_{o}(t, x)-\rho_{o} G(t, x)\right], \\
U_{w} & =N(y)\left[\nabla P_{w}(t, x)-\rho_{o} G(t, x)\right] .
\end{aligned}
$$

Учитывая эти соотношения и приравнивая коэффициенты при $\varepsilon^{0}$ в $(24)$ и при $\varepsilon$ в $(25)$, имеем

$$
\begin{gathered}
-\operatorname{div}_{y}\left(K_{f} \Lambda_{f}^{o}\left(S_{o}^{f}\right) \nabla_{y} W_{o}^{f}\right)=r_{f}^{o} G_{o}-m_{f}\left(S_{o}^{f}\right)^{\prime}+\operatorname{div}_{y}\left(\Lambda_{f}^{o}\left(S_{o}^{f}\right) K_{f} \nabla_{x} U_{o}\right)+ \\
+\operatorname{div}_{x}\left(\Lambda_{f}^{o}\left(S_{o}^{f}\right)\left(K_{f}+K_{f} \nabla_{y} N\right)\left[\nabla P_{o}^{f}-\rho_{o} G\right]\right) \\
-\left(K_{f} \Lambda_{f}^{o}\left(S_{o}^{f}\right) \nabla_{y} W_{o}^{f}, \Upsilon_{\varepsilon}\right)=\left(\Lambda_{f}^{o}\left(S_{o}^{f}\right) K_{f} \nabla_{x} U_{o}, \Upsilon_{\varepsilon}\right)- \\
-\vartheta\left(\Lambda_{b}^{o}\left(S_{o}^{b}\right) K_{b} \nabla_{y} V_{o}^{b}, \Upsilon_{\varepsilon}\right)
\end{gathered}
$$


Рассмотрим эти соотношения как задачу Неймана для $W_{o}^{f}$ с параметром $x$. Такая задача разрешима [22], если и только, если выполнено равенство

$$
\begin{gathered}
R_{f}^{o} G_{o}-M_{f}\left(S_{o}^{f}\right)^{\prime}+\operatorname{div}_{x}\left(\Lambda_{f}^{o}\left(S_{o}^{f}\right) K\left[\nabla P_{o}^{f}-\rho_{o} G\right]\right)= \\
=-\vartheta \int_{\partial Y_{f}}\left(\Lambda_{b}^{o}\left(S_{o}^{b}\right) K_{b} \nabla_{y} V_{o}^{b}, \Upsilon\right) d s
\end{gathered}
$$

Аналогично, учитывая представление $(27)$, для функций $S_{w}^{f}, P_{w}^{f}$ и $V_{w}^{b}$ получаем следующее равенство

$$
\begin{gathered}
R_{f}^{w} G_{w}-M_{f}\left(S_{w}^{f}\right)^{\prime}+\operatorname{div}_{x}\left(\Lambda_{f}^{w}\left(S_{w}^{f}\right) K\left[\nabla P_{w}^{f}-\rho_{w} G\right]\right)= \\
=-\vartheta \int_{\partial Y_{f}}\left(\Lambda_{b}^{w}\left(S_{w}^{b}\right) K_{b} \nabla_{y} V_{w}^{b}, \Upsilon\right) d s
\end{gathered}
$$

Определим осциллирующие функции нулевого порядка в разложении (20) равенствами

$$
V_{o}=\frac{Q_{o} G_{o}}{\vartheta \Lambda_{b}^{o}\left(S_{o}^{b}\right)}-\frac{Q\left(S_{o}^{b}\right)^{\prime}}{\vartheta \Lambda_{b}^{o}\left(S_{o}^{b}\right)}, \quad V_{w}=\frac{Q_{w} G_{w}}{\vartheta \Lambda_{b}^{w}\left(S_{w}^{b}\right)}-\frac{Q\left(S_{w}^{b}\right)^{\prime}}{\vartheta \Lambda_{b}^{w}\left(S_{w}^{b}\right.} .
$$

Из определений функций $Q_{o}(y), Q(y)$ и $Q_{w}(y)$, приведенных перед теоремой 2 , и формулы интегрирования по частям имеем следующие равенства

$$
\begin{aligned}
M_{b} & =\int_{Y_{b}} m_{b} d y=\int_{\partial Y_{b}}\left(K_{b} \nabla_{y} Q, \Upsilon\right) d s \\
R_{b}^{o} & =\int_{Y_{b}} r_{b}^{o} d y=\int_{\partial Y_{b}}\left(K_{b} \nabla_{y} Q_{o}, \Upsilon\right) d s \\
R_{b}^{w} & =\int_{Y_{b}} r_{b}^{w} d y=\int_{\partial Y_{b}}\left(K_{b} \nabla_{y} Q_{w}, \Upsilon\right) d s
\end{aligned}
$$

Учитывая эти равенства и определения (31) заключаем, что равенства (29) и (30) совпадают в точности с первым и вторым уравнениями осредненной начально-краевой задачи (12).

Из энергетических оценок для решений начально-краевой задачи (4), приведенных в $[1,2]$, следует, что последовательности из соотношений (13) равномерно ограничены в $L^{2}\left(0, T ; L^{2}(\Omega)\right)$, а последовательности относящиеся к объемным долям $S_{o}^{\varepsilon}$ и $S_{w}^{\varepsilon}$ ограничены в $L^{2}\left(0, T ; H^{1}(\Omega)\right)$. Таким образом, из теорем компактности, приведенных, например, в [23], следует выполнение соотношений сходимости (13).

По определению третье уравнение в задаче (4) имеет вид

$$
\chi_{f}^{\varepsilon} P_{w}^{\varepsilon}-\chi_{f}^{\varepsilon} P_{o}^{\varepsilon}=P_{f}^{c}\left(S_{w}^{f \varepsilon}\right)
$$

для множества разломов $\Omega_{f}^{\varepsilon} \times(0, T)$. Поэтому переходя в этом равенстве к пределу и учитывая предположения (9) и соотношения (13), заключаем, что выполнено третье уравнение в осредненной задаче (12).

Аналогично, четвертое и пятое уравнения в (12) следуют из четвертого уравнения в задаче (4) и соотношений (13).

Для асимптотически приближенных решений (20) имеем соотношения

$$
\chi_{b}^{\varepsilon} P_{w}^{a}=\chi_{b}^{\varepsilon} P_{w}(t, x)+V_{w}(t, x, x / \varepsilon),
$$




$$
\chi_{b}^{\varepsilon} P_{o}^{a}=\chi_{b}^{\varepsilon} P_{o}(t, x)+V_{o}(t, x, x / \varepsilon),
$$

где учтены только существенные для дальнейшего слагаемые, граничные условия для функций $Q_{o}(y), Q(y), Q_{w}(y)$ и определения (31). Таким образом, переходя к пределу в соотношениях (32) получаем утверждения (16) теоремы 2, поскольку предполагается выполнение условия (2) и приближенные решения (20) удовлетворяют уравнения из (4) для $P_{w}^{\varepsilon}$ и $P_{o}^{\varepsilon}$ с асимптотической точностью в силу приведенных построений приближений.

Для множества блоков, третье уравнение в задаче (4) имеет вид

$$
\chi_{b}^{\varepsilon} P_{w}^{\varepsilon}-\chi_{b}^{\varepsilon} P_{o}^{\varepsilon}=P_{b}^{c}\left(S_{w}^{b \varepsilon}\right)
$$

на множестве $\Omega_{b}^{\varepsilon} \times(0, T)$. Переходя к пределу в этом соотношении и учитывая соотношения (16) и (31), получаем

$$
\begin{gathered}
P_{w}-P_{o}=P_{b}^{c}\left(S_{w}^{b}\right)- \\
-\frac{\vartheta_{w}^{r} G_{w}}{\vartheta \vartheta_{b} \Lambda_{b}^{w}\left(S_{w}^{b}\right)}+\frac{\vartheta_{m}\left(S_{w}^{b}\right)_{t}^{\prime}}{\vartheta \vartheta_{b} \Lambda_{b}^{w}\left(S_{w}^{b}\right)}+\frac{\vartheta_{o}^{r} G_{o}}{\vartheta \vartheta_{b} \Lambda_{b}^{o}\left(S_{o}^{b}\right)}+\frac{\vartheta_{m}\left(S_{w}^{b}\right)_{t}^{\prime}}{\vartheta \vartheta_{b} \Lambda_{b}^{o}\left(S_{o}^{b}\right)},
\end{gathered}
$$

где учтено равенство $S_{o}^{b}+S_{w}^{b}=1$. Кроме того, используя третье равенство из (12), имеем

$$
\begin{gathered}
\frac{\vartheta_{m}\left(\Lambda_{b}^{w}\left(S_{w}^{b}\right)+\Lambda_{b}^{o}\left(S_{o}^{b}\right)\right)\left(S_{w}^{b}\right)_{t}^{\prime}}{\vartheta \vartheta_{b} \Lambda_{b}^{w}\left(S_{w}^{b}\right) \Lambda_{b}^{o}\left(S_{o}^{b}\right)}= \\
=P_{f}^{c}\left(S_{w}^{f}\right)-P_{b}^{c}\left(S_{w}^{b}\right)+\frac{\vartheta_{w}^{r} G_{w}}{\vartheta \vartheta_{b} \Lambda_{b}^{w}\left(S_{w}^{b}\right)}-\frac{\vartheta_{o}^{r} G_{o}}{\vartheta \vartheta_{b} \Lambda_{b}^{o}\left(S_{o}^{b}\right)},
\end{gathered}
$$

что совпадает с уравнением (17) и завершает доказательство теоремы 2.

Пусть выполнено условие (3). Тогда $\vartheta=\sigma / \varepsilon^{2}$ можно считать малым параметром в приведенных построениях асимптотического приближения (20). Таким образом, повторяя приведенные построения, вместо равенства (29) получаем следующее равенство

$$
R_{f}^{o} G_{o}-M_{f}\left(S_{o}^{f}\right)^{\prime}+\operatorname{div}_{x}\left(\Lambda_{f}^{o}\left(S_{o}^{f}\right) K\left[\nabla P_{o}^{f}-\rho_{o} G\right]\right)=0,
$$

которое совпадает с первым уравнением осредненной начально-краевой задачи (12) при $M_{b}=R_{b}^{o}=R_{b}^{w}=0$. Аналогично, для функций $S_{w}^{f}, P_{w}^{f}$ и $V_{w}^{b}$ вместо равенства (30) имеем соотношение

$$
R_{f}^{w} G_{w}-M_{f}\left(S_{w}^{f}\right)^{\prime}+\operatorname{div}_{x}\left(\Lambda_{f}^{w}\left(S_{w}^{f}\right) K\left[\nabla P_{w}^{f}-\rho_{w} G\right]\right)=0,
$$

которое совпадает со вторым уравнением осредненной начально-краевой задачи (12) при $M_{b}=R_{b}^{o}=R_{b}^{w}=0$.

Кроме того, учитывая в (33), что $\vartheta=\sigma / \varepsilon^{2}$ является малым параметром, получаем уравнение (19) для $S_{w}^{b}$ с точностью до малых величин, которые не являются существенными для предельного перехода при $\vartheta \rightarrow 0$.

Далее, повторяя доказательство утверждений (16), имеем соотношения

$$
\begin{gathered}
\left(\sigma / \varepsilon^{2}\right) \chi_{b}^{\varepsilon} P_{o}^{\varepsilon} \rightarrow \frac{\vartheta_{o}^{r} G_{o}}{\Lambda_{b}^{o}\left(S_{o}^{b}\right)}-\frac{\vartheta_{m}\left(S_{o}^{b}\right)_{t}^{\prime}}{\Lambda_{b}^{o}\left(S_{o}^{b}\right)}, \\
\left(\sigma / \varepsilon^{2}\right) \chi_{b}^{\varepsilon} P_{w}^{\varepsilon}-\frac{\vartheta_{w}^{r} G_{w}}{\Lambda_{b}^{o}\left(S_{w}^{b}\right)}-\frac{\vartheta_{m}\left(S_{w}^{b}\right)_{t}^{\prime}}{\Lambda_{b}^{w}\left(S_{w}^{b}\right)},
\end{gathered}
$$


которые совпадают с соотношениями (18) при выполнении условия (3), что по существу завершает доказательство теоремы 3.

Таким образом, из приведенных соотношений также следует, что ограничения давлений $P_{o}^{\varepsilon}$ и $P_{w}^{\varepsilon}$ на систему блоков $\Omega_{b}^{\varepsilon} \times(0, T)$ могут иметь асимптотически большие компоненты при малых $\sigma, \varepsilon$ и $\sigma / \varepsilon^{2}$, что эквивалентно предположению о выполнении условия (3).

Далее, из определений (20) и соотношений (23) и (32) получаем равенства

$$
\begin{gathered}
\chi_{b}^{\varepsilon} \nabla P_{w}^{a}=\chi_{b}^{\varepsilon} \nabla P_{w}(t, x)+\varepsilon^{-1} \nabla_{y} V_{w}(t, x, x / \varepsilon)+\nabla_{x} V_{w}(t, x, x / \varepsilon), \\
\chi_{b}^{\varepsilon} \nabla P_{o}^{a}=\chi_{b}^{\varepsilon} \nabla P_{o}(t, x)+\varepsilon^{-1} \nabla_{y} V_{o}(t, x, x / \varepsilon)+\nabla_{x} V_{o}(t, x, x / \varepsilon) .
\end{gathered}
$$

Следовательно, учитывая равенства (31) при $\vartheta=\sigma / \varepsilon^{2}$, заключаем, что ограничения градиентов давлений $P_{o}^{\varepsilon}$ и $P_{w}^{\varepsilon}$ на систему блоков $\Omega_{b}^{\varepsilon} \times(0, T)$ могут иметь асимптотически большие компоненты при малых $\sigma, \varepsilon$ и $\sigma / \varepsilon$, что может быть верно и при выполнении одного из условий (1)-(3), например, для $\sigma=\varepsilon^{1+\kappa}, \sigma=\varepsilon^{2}$ или $\sigma=\varepsilon^{3}$ и положительном $\kappa$.

Кроме того, в соответствии с (31), даже при фиксированных параметрах $\sigma, \varepsilon$ и $\sigma / \varepsilon$ можно локально увеличивать значения градиентов давлений на системе блоков за счет выбора функций $G_{o}(t, x)$ и $G_{w}(t, x)$ из $(12)$, которые моделируют величину и плотность внешних источников при многофазной диффузии и фильтрации в пористых средах.

Пусть выполнено условие (1). Тогда $\vartheta^{-1}=\varepsilon^{2} / \sigma$ можно считать малым параметром в приведенных построениях асимптотического приближения (20). Поэтому, из соотношения (33) получаем уравнение (15) после предельного перехода. Далее, утверждение (14) из теоремы 1 следует из определений (31) и (32), что завершает доказательство теоремы 1.

\section{ЗАКЛЮЧЕНИЕ}

В работе построены и обоснованны осредненные начально-краевые задачи, решения которых приближают решения рассматриваемой начальнокраевой задачи, моделирующей процессы многофазной диффузии и фильтрации в пористых средах. Форма таких осредненных задач зависит от выполнения одного из условий (1)-(3). В приведенных построения учитывается, что многие реальные пористые среды образованы большим количеством «блоков», имеющих слабую проницаемость, и разделенных связной системой «разломов» с высокой проницаемостью. Учет такого строения пористых сред при моделировании приводит к зависимости рассматриваемых моделей от двух параметров микромасштабности пористой среды и проницаемости блоков и усложняет вывод осредненных уравнений.

Установлены условия возникновения больших давлений и градиентов давлений на системе блоков, зависящие от величины малости параметров микромасштабности пористой среды, проницаемости блоков и некоторых отношений этих параметров. Эти условия важны при моделирования процессов многофазной диффузии и фильтрации в пористых средах, происходящих при разработке месторождений нефти и газа, поиска методов предотвращения техногенных загрязнений (нефтепродуктами) в подземных водах и нахождения способов очистки таких вод от загрязнений. 
Полученные условия также важны на практике для оптимизации таких процессов, например, за счет выбора величины и плотности внешних источников при многофазной диффузии и фильтрации в пористых средах, поскольку только с помощью больших давлений и градиентов давлений можно надеяться вытеснить и, возможно, полностью извлечь, например, нефтепродукты из системы блоков с очень слабой проницаемость.

Работа выполнена при финансовой поддержке МОН Украины (проект «Математичне моделювання та оптимізація динамічних систем для оборони, медицини та екології», 0219U008403).

\section{ЛитеРАТУРА}

1. Alt H. W. end Luckhaus S. Quasilinear elliptic-parabolic differential equations. Mathematische Zeitschrift. 1983. Vol. 183:3. P. 311-341.

2. Kroener D. end Luckhaus S. J. Flow of oil and water in a porous medium. J. Differential Equations. 1984. Vol. 55:2. P. 276-288.

3. Mandal A. and Bera A. Modeling of flow of oil-in-water emulsions through porous media. Petroleum Science. 2015. Vol. 12:2. P. 273-281.

4. Endo Kokubun M. A., Radu F. A., Keilegavlen E., Kumar K., and Spildo K. Transport of polymer particles in oil-water ow in porous media: enhancing oil recovery. Transport in Porous Media. 2019. Vol. 126. P. 501-519.

5. Bourgeat A., Luckhaus S., and Mikelic A. Convergence of the homogenization hrocess for a double-porosity model of immiscible two-phase flow. SIAM J. Math. Analysis. 1996. Vol. 27:6. P. 1520-1543.

6. Amazianea B. and Pankratov L. Homogenization of amodel for water-gas flow through double-porosity media. Math. Meth. Appl. Sci. 2016. Vol. 39. P. 425-451.

7. Sandrakov G. V. The homogenization of nonstationary equations with contrast coefficients. Dokl. Mathematics. 1997. Vol. 56:1. P. 586-589.

8. Sandrakov G. V. Homogenization of elasticity equations with contrasting coefficients. Sb: Mathematics. 1999. Vol. 190:12. P. 1749-1806.

9. Sandrakov G. V. Multiphase models of nonstationary diffusion in homogenization. Comput. Math. Math. Phys. 2004. Vol. 44:10. P. 1741-1756.

10. Sandrakov G. V. Homogenization of variational inequalities for non-linear diffusion problems in perforated domains. Izvestiya: Math. 2005. Vol. 69:5. P. 1035--1059.

11. Sandrakov G. V. Multiphase homogenized diffusion models for problems with several parameters. Izvestiya: Mathematics. 2007. Vol. 71:6. P. 1193-1252.

12. Sandrakov G. Homogenization of some hydrodynamics problems. Modern Problems of Mathematical Modeling, Computaional Methods and Information Technologies. Proceedings of the International Scientific Conf. 2-4 March 2018, Rivne. P. 156-157.

13. Sandrakov G. Modeling of hydrodynamics processes with phase transition. Information Technologies and Computer Modelling. Proceedings of the International Scientific Conf. 14-19 May 2018, Ivano-Frankivsk. P. 303-306.

14. Sandrakov G. Modeling of heterogeneous fluid dynamics with phase transition. Information Technologies in Education, Science and Technology. Proceedings of Int. Scientific-Practical Conf. 17-18 May 2018, Cherkasy. P. 142-143.

15. Sandrakov G. V. Methods of modeling of hydrodynamic processes with phase transitions. Slovak international scientific J. 2018. Vol. 1:23. P. 44-49. (In Russian) 
16. Sandrakov G. V. Modeling of phase transitions graphite-diamond. East European Scientific J. 2018. Vol. 39:11. P. 37-42. (In Ukrainian)

17. Sandrakov G. V. Modeling and homogenization of hydrodynamics processes with the oscillating data. East European Scientific J. 2018. Vol. 40:12. P. 56-62. (In Ukrainian)

18. Sandrakov G. Modeling and homogenization of hydrodynamics processes with the vanishing viscosity. Int. School-Workshop on Differential Equations and Applications. 18-20 June, 2019, Vinnytsia. Book of Abstracts. p. 62-63.

19. Sandrakov G. V., Lyashko S. I., Bondar E. S., Lyashko N. I. Modeling and optimization of microneedle systems. J. Automation and Information Sciences. 2019. Vol. 51:6. P. 1-11.

20. Sandrakov G. Homogenized models with memory effects for composites. Int. School-Workshop on Differential Equations and Applications. 18-20 June, 2019, Vinnytsia. Book of Abstracts. P. 63-64.

21. Bensoussan A., Lions J.-L., and Papanicolau G. Asymptotic analysis for periodic structures. North-Holland, Amsterdam 1978.

22. Bakhvalov N. S. and Panasenko G. P. Homogenization: averaging processes in periodic media. Kluwer, Dordrecht 1989.

23. Duvaut G. end Lions J.-L. Les inequations en mecanique et en physique. Dunod, Paris 1972.

Поступила: 30.10.2019 / Принята: 07.11.2019

\section{ОСЕРЕДНЕНІ МОДЕЛІ БАГАТОФАЗНОЇ ДИФУЗІЇ В ПОРИСТИХ СЕРЕДОВИЩАХ}

\section{Г. В. САНДРАКОВ}

Факультет комп'ютерних наук і кібернетики, Київський національний університет імені Tараса Шевченка, Київ, Україна, E-mail: gsandrako@gmail.com

АнотАція. Розглядаються нестаціонарні процеси взаємної дифузії багатофазних потоків рідин, які не змішуються між собою, в пористих середовищах з періодичною структурою. Математичною моделлю для таких процесів є початково-крайова задача дифузії для середовищ, утворених великою кількістю «блоків», що мають слабку проникність, і розділених зв'язковою системою «розломів» з високою проникністю. Прийняття до уваги такої будови пористих середовищ призводить до залежності рівнянь цієї задачі від двох малих параметрів мікромасштабності пористого середовища та проникності блоків. Будуть отримані усереднені початково-крайові задачі, розв'язки яких наближають розв'язки даної початково-крайової задачі.

КЛЮчові СловА: багатофазна дифузія, процеси фільтрації, пористі середовища, моделювання, початково-крайові задачі, осереднені моделі. 